\title{
Framework para la evaluación de calidad de procesos ágiles
}

\section{Framework for the quality assessment of agile processes}

Presentación: 06/10/2020

\section{Doctoranda:}

\section{Noelia Soledad Pinto}

Centro de Investigación Aplicadas a Tecnologías de la información y la comunicación (CInApTIC), Universidad Tecnológica Nacional, Facultad Regional Resistencia - Argentina

ns.pinto@gmail.com

\section{Director:}

\section{César J. Acuña}

\section{Co-director:}

\section{Gustavo Rossi}

\section{Resumen}

La industria del software requiere de productos y servicios de alta calidad, puede lograrse mediante la aplicación de modelos y metodologías de calidad reconocidos internacionalmente. Sin embargo, estos modelos en pequeñas y medianas empresas (PYMES) son muy difíciles de implementar ya que ello implica una gran inversión en dinero, tiempo y recursos.

Por ello, resulta necesario establecer estrategias que permitan la automatización del ciclo de desarrollo de software, de forma tal de promover la reducción de costos y la eficiencia en los procesos de obtención de productos finales. En este caso, la filosofía ágil resulta ser el enfoque más adecuado para los entornos de desarrollo actuales, y se posicionan como alternativa a los procesos de desarrollo con alto costo en documentación y procesos excesivamente prolongados.

Con el objetivo de facilitar la adopción de prácticas ágiles en las PYMES, promoviendo el aseguramiento de la calidad de los procesos de desarrollo de software, se presenta Agile Quality Framework (AQF), un framework cuya propuesta integra el diseño y desarrollo de un modelo que permita evaluar la calidad en procesos ágiles de software, y una plataforma que permitirá el seguimiento de proyectos de software ágiles junto a la evaluación sistemática de calidad del proceso de desarrollo.

AQF surge, como una plataforma que contribuye con los equipos de desarrollo de software a partir de la evaluación de calidad en proyectos ágiles, considerando como objeto de la medición al proceso de desarrollo independientemente del enfoque ágil seleccionado.

Palabras clave: Calidad, Software, Agilidad

\footnotetext{
Abstract

The software industry requires high-quality products and services, which is achieved through the application of internationally recognized quality models and methodologies. However, these models in small and medium-sized companies (SMEs) are very difficult to implement due to high cost, long time and specialized human resources.
} 
Therefore, it is necessary to establish strategies that allow the automation of the software development cycle, in such a way as to promote cost reduction and efficiency in the processes for obtaining final products. In this case, the agile philosophy turns out to be the most appropriate approach for current development environments, and they are positioned as an alternative to development processes with high cost in documentation and excessively long processes.

With the aim of facilitating the adoption of agile practices in SMEs that ensure the quality of software development processes, the Agile Quality Framework (AQF) has been presented, a framework whose proposal integrates the design and development of a model that allows evaluating quality in processes agile software, and a web application that will allow the monitoring of agile software projects together with the systematic evaluation of the quality of the development process.

AQF emerges as a platform that contributes to software development teams from the quality assessment in agile projects, considering the development process as an object of measurement regardless of the agile approach selected.

Keywords: Quality, Software, Agile

\section{Introducción}

La mejora e innovación de los procesos de software, con el objetivo de incrementar la calidad de sus productos y servicios, se ha convertido, en los últimos años, en el elemento diferenciador que las empresas necesitan para mejorar sus niveles de competitividad en la Industria del Software. Sin embargo, diversos estudios coinciden en la dificultad de las PYMES (Mas., Amengual., 2005)( Pasini, Esponda, Bertone \& Pesado, 2008) (Pflegger, 2002) para implementar programas de Mejoras de Proceso de Software (Software Process Improvement - SPI), fundamentalmente porque la aplicación de estos modelos resulta costosa en términos económicos y de esfuerzo, pues requieren una gran inversión en dinero, tiempo y recursos, sus recomendaciones son complejas de aplicar y el retorno de la inversión se produce a muy largo plazo (Garzás, Fernández \& Piattini, 2009). De esta forma, los parámetros de tiempos de desarrollo y costo de soluciones afectarán directamente al trabajo que se realice, siendo la calidad la primera variable de ajuste disponible.

Dado que la calidad del producto de software desarrollado está estrechamente relacionada con la calidad del proceso utilizado, las PyMEs necesitan implementar proyectos para la mejora de sus procesos que le permitan incrementar la calidad de sus productos. De esta forma, analizando la situación de la Industria del Software en el NEA (Región Nordeste de Argentina) respecto a la adopción del ciclo de vida que guíe los procesos de desarrollo de las empresas, surge la necesidad de proporcionar un marco de trabajo que permita evaluar la calidad cuando optan por trabajar con enfoques ágiles (Acuña, Cuenca Pletsch, Tomaselli, Pinto \& Tortosa; 2015).

Habiendo realizado un análisis de la literatura disponible se observa que, en su mayoría, los estudios se centran en analizar la implementación de prácticas ágiles, sin evaluar la relación entre el proceso de desarrollo de software y la calidad. Así, y teniendo en cuenta las particularidades de organizaciones tipo PYMES, resultados de estudios previos realizados (Rujana, Romero Franco, Tortosa, Tomaselli, \& Pinto, 2016), demuestran la ausencia de estrategias que permitan a las empresas integrar agilidad a sus ciclos de desarrollo sin dejar de lado aspectos relacionados a la calidad de software.

En este sentido, y como primera alternativa, se viene trabajando en la implementación y validación de un marco de trabajo que permita evaluar la calidad cuando se opta por trabajar con procesos ágiles de desarrollo de software. Dicho framework se denomina AQF (Agile Quality Framework) y su versión actual está formada por un modelo, QuAM (Quality Agile Model), compuesto por métricas, atributos y criterios que permiten medir los niveles de calidad asociados a las prácticas ágiles, y por QuAGI (Quality AGIle), una herramienta de software que brinda soporte a dicho modelo a través de la automatización del seguimiento de proyectos y la visualización de diferentes informes (Pinto, Acuña, Tortosa \& Cabas Geat; 2017). Cabe destacar que este desarrollo constituye uno de los objetivos de la tesis doctoral denominada "Framework para la evaluación de calidad de 
procesos ágiles", realizada en el marco del proyecto "Evaluación de Calidad en Procesos Ágiles de Desarrollo de Software", financiado por la UTN y ejecutado en el Centro de Investigación aplicada en TICS (CInApTIC) de la Facultad Regional Resistencia, con el código IAI4445TC.

A continuación, en la sección Desarrollo de este artículo, se presenta AQF, sus características principales y los resultados más relevantes luego de diversas experiencias de validación. Luego se exponen conclusiones junto a desafíos futuros de investigación, derivados de la realización de esta tesis doctoral.

\section{Desarrollo}

El objetivo principal de la tesis doctoral es proponer un framework que facilite el seguimiento de proyectos y la evaluación de calidad de procesos cuyos ciclos de desarrollo implementan prácticas ágiles. El aporte significativo de AQF se centra en que el nivel de calidad de un proyecto ágil no sea definido únicamente a través de un valor numérico, sino que sea el resultado también de la evaluación de otros aspectos, en base al contexto en el que se desarrolla el proceso y los factores que lo impactan. Aquí, es necesario aclarar que, cuando se hace referencia al término "Proyecto Ágil", se incluye a aquéllos que implementan prácticas ágiles para el proceso de desarrollo del producto software en base a cualquier enfoque tal como Scrum, Kanban, Lean o híbridos.

\section{AQF: Características del Framework}

AQF está compuesto por:

- Un modelo de calidad, denominado QuAM (Quality Agile Model) que consta de:

- Una estructura formal definida en función a componentes, atributos, métricas y criterios relacionados entre sí.

- Una estrategia de medición que establece el nivel de calidad asociado a cada componente.

- Una herramienta de software, QuAGI (Quality AGIle) que da soporte al modelo, constituyendo un medio para su validación, y se compone de:

○ Una arquitectura que define cada uno de los componentes que forman la herramienta.

○ Un proceso de medición sobre cada uno de los componentes del modelo QuAM.

○ Un conjunto de interfaces que facilitan la interacción de actores externos (administrador del proyecto, equipo de desarrollo, etc) con la herramienta.

QuAM (Quality Agile Model), es un modelo conceptual cuyo objetivo es describir el conjunto de componentes que influye en la calidad de proyectos ágiles junto a un procedimiento de evaluación de calidad que los integra. QuAM permite asociar los resultados de la medición con un perfil de calidad asociado al proyecto ágil evaluado (Pinto, Tomaselli, Acuña, Cuenca Pletsch \& Tortosa; 2016). El modelo QuAM surge de un relevamiento sistemático de la literatura y del análisis del contexto actual a partir del relevamiento de información realizado en la Industria del software en el nordeste argentino (NEA) del cual se han obtenido factores de relevancia que fueron incluidos al esquema de componentes de QuAM. Tal como se observa en la Figura 1, la estructura del modelo incluye 4 componentes, formados por atributos, cada uno de los cuales se mide a través de métricas directas e indirectas.

En QuAM, los componentes representan a cada uno de los aspectos que impactan en la calidad del proceso de desarrollo de software y que componen el proyecto ágil a evaluar. Cada uno de estos componentes se caracteriza por atributos, los cuales representan propiedades que son de interés para fines de medición. El conjunto de atributos de cada componente puede clasificarse como: Atributos Positivos, aquéllos que se desean enfatizar y promover para aumentar los niveles de calidad, y Atributos Negativos, aquéllos que se deberán minimizar para que no afecten disminuyendo los niveles de calidad del proceso. Asimismo, un atributo puede ser cuantificado por una o varias métricas, dependiendo de cómo se realice la medición. En la definición de QuAM, la evaluación de cada componente se especifica a través del proceso de medición de las métricas asociadas a los atributos que lo componen, cuyo resultado ofrece evidencia empírica para poder comprender, de mejor manera, las diferentes dimensiones que engloban la calidad del proyecto ágil que se analice. 


\begin{tabular}{l}
\hline Componente 1: Capacidad de Producción de Entregables \\
- Atributo Positivo: Valor a la estimación \\
- Atributo Negativo: Valor al Lead Time \\
Componente 2: Evaluación del Equipo de Trabajo \\
- Atributo Positivo: Valor a la definición de roles y tareas \\
- Atributo Negativo: Valor al proceso por sobre el equipo \\
Componente 3: Comunicación con el cliente \\
- Atributo Positivo: Valor a la colaboración con el cliente \\
- Atributo Negativo: Valor a la negociación contractual \\
Componente 4: Gestión de Requerimientos \\
- Atributo Positivo: Valor a la completitud de requerimientos \\
- Atributo Negativo: Valor a la dependencia entre requerimientos
\end{tabular}

Figura 1. Componentes y atributos del modelo de calidad QuAM

Sin embargo, para la evaluación de calidad de proyectos ágiles que propone AQF no basta con la definición de un modelo, sino que resulta necesario automatizar la gestión de los componentes de QuAM a partir de una herramienta que permita el seguimiento de proyectos acompañado por la obtención de informes parciales respecto de la calidad del proceso ágil que lo caracteriza. Por ello, a fin de complementar el framework AQF, se aborda el desarrollo de QuAGI, una aplicación web que permite la gestión de los componentes de QuAM a través de la medición y obtención automática de los valores correspondientes a cada una de las métricas a lo largo del proceso de desarrollo. El objetivo central de esta herramienta es permitir el seguimiento online de proyectos basados en prácticas ágiles junto a la posibilidad de realizar evaluaciones continuas respecto al nivel de calidad que se esté logrando en el proceso.

QuAGI permite administrar proyectos ágiles, realizar el seguimiento de cada uno de ellos, obtener diversos informes y constituye, además, una herramienta de comunicación interna entre los integrantes del equipo. Por último, da soporte a los procesos de toma de decisiones asistiendo a los responsables mediante reportes que informen sobre evaluación de calidad del proyecto y recomendaciones de ajustes para la mejora continua (Pinto, Tortosa, Cabas Geat, Ibáñez \& Acuña; 2018).

La arquitectura propuesta para QuAGI consiste en una plataforma basada en componentes reutilizables, permitiendo así que las aplicaciones que se integren a la plataforma hagan uso de dichos componentes. Tal como se observa en la Figura 2, cada uno de los componentes de QuAGI representa una vista particular de su arquitectura, y esta configuración propuesta permite asegurar el mantenimiento de la trazabilidad entre los diferentes artefactos desarrollados, la reutilización de estos y el mejor control de su evolución a la hora de incorporar nuevas funcionalidades.

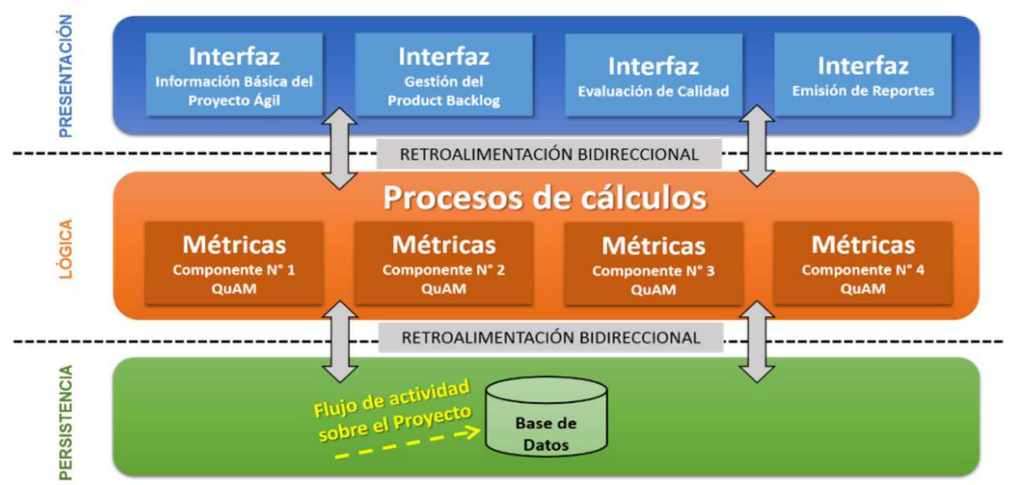

Figura 2. Arquitectura conceptual de QuAGI

Considerando que existen actualmente en el mercado muchas herramientas que permiten gestionar proyectos de software (por ejemplo, Trello, Taiga.io, Jira, etc), QuAGI, a diferencia de las existentes, además forma parte del framework AQF, por lo 
que no solo permite el seguimiento online de los proyectos de software, sino que también da soporte a lo establecido en un modelo de calidad propio y permite, entonces, realizar evaluación de calidad del proyecto en cuestión. Así, actualmente QuAGI permite: Configuración inicial del proyecto ágil, Gestión del Product Backlog, Evaluación de calidad y Emisión de reportes.

Cabe destacar que QuAGI almacena constantemente la interacción de los usuarios con la herramienta; por lo que, cuando se requiere conocer sobre el nivel de calidad del proyecto, QuAGI recupera dicha información, realiza las mediciones de todos los componentes, definidos por QuAM, y obtiene la evaluación de forma automática. Una de las contribuciones más importantes derivadas de esta tesis se enfoca en la posibilidad de incorporar, al seguimiento de proyectos ágiles, el proceso de evaluación de calidad asociado al ciclo de desarrollo que se pretende gestionar.

Finalmente, resulta importante destacar que, como resultado del desarrollo de esta plataforma web QuAGI la misma ha sido registrada, durante el desarrollo de la tesis, como Obra Inédita de Software ante la Dirección Nacional de Derecho de Autor dependiente del Ministerio de Justicia y Derechos Humanos de la República Argentina ${ }^{1}$.

\section{Validación}

Luego de la implementación de AQF, se ha impulsado la validación del modelo QuAM a partir del estudio de casos caracterizados por proyectos reales que han utilizado QuAGI como herramienta de seguimiento y soporte para la evaluación de calidad del proceso de desarrollo basado en prácticas ágiles.

Por un lado, luego de haberse ejecutado todos los estudios de casos se destaca cómo gracias al uso de QuAGI y sus recomendaciones, se logra mejorar la calidad de los procesos ágiles de desarrollo de software en cada proyecto. El grupo de participantes en la experiencia subrayan, además, entre los beneficios de QuAGI, que sea una herramienta fácil de usar, útil y muestran intención de usarla en el futuro, gracias a la información que han obtenido. Por otro lado, la realización de esta experiencia de validación ha permitido, además, analizar el grado de adecuación, completitud y corrección de los resultados en los informes de calidad aportados por QuAGI; logrando mejoras que aporten valor a la herramienta en cada instancia de validación.

Y, por último, pero no menos importante, el estudio de casos sobre proyectos ágiles reales ha posibilitado obtener realimentación de los participantes que aplicaron el modelo y usaron la herramienta. Eso no solo colaboró con mejoras a nivel de interfaz y usabilidad de la herramienta QuAGI, sino que también permitió añadir valor a través de mejoras en la configuración de los componentes de QuAM.

\section{Conclusiones}

La tesis doctoral que se presenta en este artículo tiene como objetivo principal el diseño, desarrollo e implementación del framework, AQF, para la evaluación de calidad en procesos ágiles de desarrollo de software.

El trabajo de esta tesis ha permitido integrar en AQF la presentación de un nuevo Modelo de Calidad, QuAM; y la implementación de una herramienta de software, QuAGI, que da soporte automático a lo establecido en QuAM. Y, mejor aún, la propuesta ha sido adaptada, en base a estudios de casos de validación, para que pueda utilizarse en entornos reales de producción con proyectos de software cuyo desarrollo es guiado por prácticas ágiles.

Como trabajos futuros se pretende llevar a cabo más casos de estudio que permitan lograr la versión que se adecúe, en mayor medida, a la realidad de las PyMES, incorporando sus prácticas más comunes y permitiendo obtener el nivel de calidad más

\footnotetext{
${ }^{1}$ El Registro se ha publicado en el Boletín Oficial del Poder Ejecutivo Nacional el 25 de junio de 2019, con el Número RE-201957162220-APN-DNDA-\#MJ
} 
representativo a cada proyecto ágil que se evalúe a través de AQF. Además, resulta necesario incorporar al estudio de validación el resto de los componentes para analizar el funcionamiento integral del framework.

Además, a pedido de las empresas, se continuará expandiendo AQF mediante la incorporación de un Asistente Virtual que actúe en función a eventos, libere de trabajo de monitorización a los Administradores de Proyecto y de soporte a la toma de decisiones de directivos de las empresas.

Finalmente, cabe destacar que la tesis, realizada en el marco del Doctorado en Ciencias Informáticas de la UNLP, se encuentra en proceso de evaluación, con una fecha de lectura estimada en los próximos 2 meses. Y, como resultado de su realización, se han generado 2 proyectos de investigación, "Evaluación del impacto de las emociones en la calidad de software desde el punto de vista del usuario", aprobado y en ejecución, y otro, en evaluación, denominado "iQuAGI: Un enfoque inteligente para la evaluación de calidad de procesos de software ágiles”, ambos pertenecientes al CInApTIC.

\section{Referencias}

Acuña, C., Cuenca Pletsch, L., Tomaselli, G., Pinto, N., Tortosa, N. (2015) "Calidad de Software y Metodologías Ágiles en las PYMES de la Industria del Software". Publicado en Memorias de 3er Congreso Nacional de Ingeniería Informática / Sistemas de Información (CONAIISI 2015). Noviembre, 2015. ISBN 978-987-1896-47-9.

Garzás, Fernández \& Piattini (2009). "Una aplicación de la Norma ISO/IEC 15504 para la evaluación por nivel de madurez de Pymes y pequeños equipos de desarrollo". Revista Española de Innovación, Calidad e Ingeniería del Software, Vol.5, No. 2.

Mas A., Amengual E. (2005). "Las mejoras de los procesos de Software en las pequeñas y medianas empresas (pymes). Un nuevo modelo y su aplicación a un caso real". Revista Española de Innovación, Calidad e Ingeniería del Software, Vol.1, No. 2

N. Pinto; C. Acuña; N. Tortosa; B. Cabas Geat (2017) XXIII Congreso Argentino de Ciencias de la Computación. La Plata, Buenos Aires. "Evaluating Quality in Agile Developments. A first validation experience with NEA Software SMEs."

Noelia Pinto; Gabriela Tomaselli; César J. Acuña; Liliana Cuenca Pletsch; Nicolás Tortosa (2016). Hacia un modelo de evaluación de calidad de procesos ágiles. CONAIISI 2016. ISSN 2347-0372.

Pasini, A. C., Esponda, S., Bertone, R. A., \& Pesado, P. (2008). "Aseguramiento de Calidad en PYMES que desarrollan software." XIV Congreso Argentino de Ciencias de la Computación.

Pflegger, S. (2002) "Ingeniería de Software. Teoría y Práctica." Pearson Education.

Pinto, N., Tortosa, N., Cabas Geat, B., Ibáñez, L., \& Acuña, C. (2019). "Validation of the reengineering applied on the first version of Agile". Electronic Journal of SADIO (EJS), 18(1), 93-109. Marzo 2019.

Rujana, M., Romero Franco, N., Tortosa, N., Tomaselli, G., \& Pinto, N. (2016). "Análisis sobre adopción de metodologías ágiles en los equipos de desarrollo en pymes del NEA". WICC 2016 